\title{
Assessment of gynaecological and general history parameters inpregnant women in long term refugee camps in Greece: results from a pilot program
}

\author{
Vasilios Pergialiotis, Chryssoula Botsi, loanna Papari, Stavroula Gkritziou, Kassiani Mellou, \\ S. Pappas, Agis Terzidis, Theofilos Rosenberg
}

Hellenic Centre for Disease Control and Prevention (KEELPNO), Athens, Greece.

Correspondence

Vasilios Pergialiotis, pergialiotis@yahoo.com

\section{Abstract}

Objective:The purpose of the present article is to present preliminary data related to the gynecological history and current health status of pregnant refugees that reside in long-term refugee camps in Greece. Materials and Methods: The study was funded by the European program "PHILOS - Emergency health response to refugee crisis" of the Greek Ministry of Health, implemented by the Hellenic Center for Disease Control and Prevention (HCDCP), funded by the Asylum, Migration and Integration Fund (AMIF) of EU's DG Migration and Home Affairs. Refugee pregnant women who resided in long-stay refugee camps of the mainland in Greece in 2017 were recruited. Results: Overall, 456 pregnant women were enrolled in the present study with a mean age of $27.5 \pm 6.9$ years (median 27, range 17-48 years). 182 women (39.8\%) were primigravidae. Mean gravidity was 2.4 (median 2, range 1-11). Among them 105 had a previous delivery, 75 women had 2 previous deliveries, 50 women had 3 previous deliveries and 14 had four previous deliveries. Of those, only six women had access to prenatal screening.Twenty-three women (5\%) reported that had at least an artificial abortion performed (median 1, range 1-4) and 87 women (19.0\%) had at least one spontaneous abortion (median 1, range 1-6).Two hundred and three women (44.5\%) reported the use of a contraceptive method. Among them withdrawal (pull-out method) was the most prevalent accounting for almost half cases (36.9\%), by followed by condom, intrauterine devices (IUD) and oral contraceptives. No differences were observed after checking the potential differences among the different age groups ( $\mathrm{p}=.234)$. Conclusions: Refugee women that reside in long term refugee camps have limited access to national healthcare systems concerning their gynecological and obstetrical assessment. Future studies, should specifically target these problems to help structure specific healthcare plans that will ensure maternal and fetal well-being.

Key words: Refugees, women, Greece, gynecological assessment, antenatal care 


\section{Introduction}

A long-run history of migration and Greece since independence (1832) can be characterized as predominantly one of emigration - of Greeks for economic reasons, and of ethnic and other minorities for political reasons. The first important immigration inflows were during and after the disastrous GrecoTurkish war of 1919-1922 and its final resolution with the Treaty of Lausanne ${ }^{1}$ This resulted in the "Exchange of Populations" with Turkey and the displacement of over 2 million persons - Christians to Greece and Muslims to Turkey. During the last years, migration flows towards Europe are at an all-time high, and provision of coordinated and appropriate health care for receiving countries, such as Greece, pose an enormous challenge ${ }^{2,3}$. In March 2016, the northern borders of Greece closed and the country had to deal with an unprecedentedly seen situation. Greece, from a previously mainly transit country, turned into one of medium- and long-term stay country with a large number of hosted refugees and migrants ${ }^{4}$. For the second time in her history, long term accommodation camps for hosting refugees and migrants and reception and identification centers for newly arrived population were established and medical services were offered inside hosting facilities. Standard operating procedures were not available and a lot of different partners with different approaches, tools and priorities acted at the field.

Overcrowding and poor personal hygiene at hosting facilities as well as limited access to health care services were the main challenge for this transit period. As the months pasted and the situation as well as the flows were stabled the medical authorities of the country had to move from emergency response to holistic approach of the refugee and migrant population of the country and implement a more integrated and individualized health care provision methods.

This was the main goal of the European program "PHILOS - Emergency health response to refugee cri- sis" of the Greek Ministry of Health, implemented by the Hellenic Center for Disease Control and Prevention (HCDCP), funded by the Asylum, Migration and Integration Fund (AMIF) of EU's DG Migration and Home Affairs. The program started in January 2017 and was completed in August 2018. Assessment of the health and of health needs of vulnerable populations, such as pregnant women living inside camps was one of the main priorities of the program.

Refugee women have been reported to be a particularly vulnerable group which merits close evaluation $^{5}$. Despite the fact that several conditions have been discussed in refugee populations the last decade including vaccination status, respiratory, gastrointestinal, chronic and mental conditions ${ }^{6}$, the gynecological assessment and treatment of female refugees remains relatively unexplored. Therefore, information related to gynecological history including menstrual characteristics, use of contraception, screening for STD`s and cervical cancer and pathologic conditions that may influence the pregnancy course is relatively limited in this specific population.

Pregnant women represent a suitablepopulation to screen for the presence of various diseases as they often seek medical help during the pregnancy course. The purpose of the present article is to present preliminary data related to the gynecological history of pregnant refugees that reside in long-term refugee camps in Greece. These data will be used to provide guidance for further research in this field that is essential to enhance vigilance, increase the detection of gynecological diseases and, ultimately, improve gynecological health status of these women.

\section{Materials and Methods}

Refugee pregnantwomen who resided in long-stay refugee camps of the mainland in Greece in 2017 were recruited. The study is in agreement with the 1964 Declaration of Helsinki and its later amendments. All 
women participated in the present study through their regular assessment by trained midwives on camp site.

All information was collected via personal interviews of the women and review of their medical records. The general recording form included the name, fathers name and age of women, a short family history of first-degree relatives related to breast and gynecological cancer as well as other medical conditions. The patient's general personal history included the presence of thyroid disease, hypertension, family history of cancer, heart disease, depression, hormone intake, HBV and or HCV seropositivity, illicit drug abuse, smoking habits, history of epilepsy, anemia, diabetes mellitus andchildhood vaccination. Specific assessment of gynecological procedures, pap smear status and history of sexually transmitted diseases (STDs) including human immunodeficiency viruswas undertaken. Extraction of data related to current menstrual status (duration of menstrual cycle, oligo- polyamenorrhea and menorrhagia), polycystic ovarian syndrome, age at menarche and age at menopause as well as parity and gravidity was also performed. Current and previous contraceptive methods were evaluated.The number of deliveries was also reported with specific stratification for operative vaginal deliveries and cesarean section (emergent/planned). The number of first and second trimester miscarriages was extracted as well as the number of previous abortions. High risk pregnancies and pregnancy complications were also evaluated.

Information was recorded with the use of a structured form and data were recorded in a specially designed database. Analysis of data was carried out with the use of the statistical package SPPS (IBM Corp. Released 2015. IBM SPSS Statistics for Windows, Version 23.0. Armonk, NY: IBM Corp.). Analysis was performed after stratifying women as adolescent, average maternal age and advanced maternal age according to the WHO`s criteria. Specifically, adolescent gravidae were considered as those that were pregnant before the completion of the 19th year of life, advanced maternal gravidae as those that were pregnant above the 35 th year of life and women between 19 and 35 years as average maternal age gravidae. An association was considered statistically significant when $p \leq 0.05$; all calculated p-values were two-tailed.Associations between quantitative variables were tested with the Student's t-test (for normally distributed variables), after checking for homoskedacity, or the Mann-Whitney test for skewed variables. Associations between continuous variables were tested with Spearman's test (not normally distributed variables).

\section{Results}

Overall, 456 pregnant women were enrolled in the present study with a mean age of $27.5 \pm 6.9$ years (median 27, range 17-48 years). The majority of women was of average maternal age (301 women) whereas seventy-two women were adolescents at time of conception and 68 women were at advanced maternal age.Only six women had access to prenatal screening of chromosomal and/or anatomical abnormalities with second trimester screening. Eighteen women received iron supplementation during the pregnancy course and among those eleven women also received folic acid supplementation. During the study period there were six first trimester spontaneous abortions, five artificial abortion, and five intrauterine deaths.

The mean age at menarche was $13.9 \pm 3.3$ years (median 13, range 11-22 years).The duration of their menstrual cycle was $26.0 \pm 8.9$ days (median 28, range 12-60 days) and of their menstruation $4.7 \pm 2.1$ days (median 5, range 2-14). Sixty women suffered from menstrual disorders, including polymenorrhea (intervals of $<20$ days) (48 women), oligomenorrhea (intervals of $>35$ days) (10 women) and amenorrhea ( $>3$ lost menstrual periods) ( 5 women).

Two hundred and three women $(44.5 \%)$ reported the use of a contraceptive method. Among them 
withdrawal (pull-out method) was the most prevalent accounting for almost half cases (36.9\%), by followed by condom, intrauterine devices (IUD) and oral contraceptives.No differences were observed after checking the potential differences among the different age groups $(\mathrm{p}=.234)$.

One hundred and eighty-two women (39.8\%) were primigravidae. Mean gravidity was 2.4 (median 2, range 1-11) (Figure 1). Among them 105 had a previous delivery, 75 women had 2 previous deliveries, 50 women had 3 previous deliveries and 14 had four previous deliveries. The correlation of age with gravidity was positive (Spearman`s $\mathrm{rho}=.253$, $\mathrm{p}<.001$ ).

Twenty-three women (5\%) reported that had at least an artificial abortion performed (median 1, range 1-4) and 87 women (19.0\%) had at least one spontaneous abortion (median 1, range 1-6).

\section{Discussion}

In 2016 the migrant crisis escalated as 173,450 arrivals of refugees were reported in Greece according to the refugee portal of the United Nations High Commissioner for Refugees (UNHCR)7. In 2017 29,718 refugees arrived in Greece by sea, most of them from the Syrian Arab Republic and Afghanistan ${ }^{7}$.

Health problems at newly arrived migrants and refugees have already been reported by Pavli and Maltezou ${ }^{6}$. Multiple conditions that include lack of communication, lack of referral systems and SOPs, cultural barriers, as well as the lack of adequate number of cultural mediators on site and at the hospitals result at low health care utilization in the population living at long term hosting camps ${ }^{8}$. This finding stresses the need for an integrated approach based on a multidisciplinary team setting in order to improve the access of the population to health care services. Pregnant women consist a vulnerable population group ofthe population with an increased risk for adverse pregnancy outcomes and to date, data concerning the gy- necological and obstetrical history of women that reside in camps are scarce and limited in the international literature ${ }^{9-11}$.

Our study provides data drawn from the basic gynecological assessment of female refugees that live in long-term refugee camps. The accumulated evidence underline a number of issues that have to be taken into account when conducting cohort studies and randomized trials and during the design of healthcare facilities to screenthis specific population.

According to the findings of our study, these women give birth during the third decade of life and the mean age at delivery seems to increase sequentially reaching approximately 29 years. To date, their access to healthcare facilities and antenatal surveillance is limited; hence, basic parameters such as antenatal screening for chromosomal and anatomic abnormalities, routine evaluation of fetal well-being and maternal iron and folate supplementation are seldom performed. Consequently, anemia is present in a significant percentage of this population.

Screening for cervical cancer with the use of pap smear was particularly limited among refugee women in our series. However, these data are consistent with other studies in non-migrant Middle Eastern Arab populations ${ }^{12,13}$. Lack of adequate information, ethnical and ethical reasons have been reported as barriers to the adoption of this health policy in women from the Middle East. However, these barriers seem to seize in migrant populations of Arab populations ${ }^{14}$, denoting the importance of a health education program to enhance awareness and improve adoption of cervical cancer screening.

Birth control measures were highly adopted in the present series of women. However, most of them utilized the pull-out method which is not very efficient due to lack of the contraceptive methods used in their countries. Barrier options, primarily male condoms, could provide an excellent cost-effective alternative that could also limit the possibility of STDs, urinary 
tract infections and genital tract infections in this setting. Artificial abortions were relatively limited in the present population. However, the expectancy of family planning should be further assessed in refugee women that reside in long term camps as living conditions in this setting are not conducive to bringing up children.

Previous studies have investigated the psychological impact of pregnancy and motherhood among immigrants and refugees and underlined several problems that provoke anxiety and refusal of further family planning among these women ${ }^{15}$. Syrian refugee women seem to suffer from depressive symptomatology and this effect seem to be exaggerated during the puerperium ${ }^{16-18}$. In our study refugee women had comparable rates of depressive symptomatology to those of previous studies ${ }^{15}$, although the actual prevalence of depression should be screened thoroughly with the use of specific test batteries for depression.

The fact that most of the collected information was self-reported and thatthe information on the exact time of arrival of women at the country was not recorded are limitations of our study. However, since information was acquired only from long term accommodation camps of the mainland (and not from the reception and identification centers of the islands and Evros where refugees are usually hosted before they are transferred to the mainland) we believe that most women conceived after their arrival at the country or recently before that. Lack of available data regarding health status / results of testing was an important limitation of our study but at the same time was a main finding that antenatal care of pregnant women was limited.

\section{Implications for future research}

Screening of female refugees that reside in long term camps is of utmost importance, as several obstetrical and gynecological issues may arise in this specific population. Gynecological assessment for the detection of sexually transmitted diseases, signs of urogenital infection and screening for cervical pathology should be planned and, ideally, specific targets should be set, taking in mind the particular ethnical and social aspects as well as the ethical beliefs of these women. To achieve this, local health policy providers should structure educational programs, taking in mind the aforementioned parameters, and evaluate women's disbeliefs and personal barriers.

\section{Conclusion}

According to the findings of our study, refugee women that reside in long term refugee camps have limited access to national healthcare systems concerning their gynecological and obstetrical assessment. Future studies, should specifically target these problems to help structure specific healthcare plans that will ensure maternal and fetal well-being.

\section{Funding}

The present article is part of the program "PHILOS - Emergency health response to refugee crisis" which was funded by the Asylum, Migration and Integration Fund (AMIF) of EU's DG Migration and Home Affairs.

\section{Ethics Aproval}

All procedures performed in studiesinvolving human participants were in accordance with theethical standards of the institutional and/or nationalresearch committee and with the 1964 Helsinki declaration and its later amendments or comparableethical standards

\section{Informed consent}

Informed consent wasobtained from all individual participantsincluded in the study.

\section{References}

1. Baldwin-Edwards. M, Apostolatou. K. 'Foreword. Ethnicity and migration: A Greek story', Migrance, 31, 1-17. 2008. 
2. Puchner K, Karamagioli E, Pikouli A, et al. Time to Rethink Refugee and Migrant Health in Europe: Moving from Emergency Response to Integrated and Individualized Health Care Provision for Migrants and Refugees. Int J Environ Res Public Health 2018;15(6).

3. Efird JT, Bith-Melander P. Refugee Health: An Ongoing Commitment and Challenge. Int J Environ Res Public Health 2018;15(1).

4. United Nations High Commissioner for Refugees (UNHCR). Refugees/Migrants Emergency Response - Mediterranean / Greece (data in excel file). [Internet]. [Accessed 30 Dec 2016]. Available from: http://data.unhcr.org/mediterranean/country.php?id=83.

5. Malebranche M, Nerenberg K, Metcalfe A, Fabreau GE. Addressing vulnerability of pregnant refugees. Bulletin of the World Health Organization 2017;95(9):611-A.

6. Pavli A, Maltezou H. Health problems of newly arrived migrants and refugees in Europe. J Travel Med 2017;24(4).

7. Read CP, Word RA, Ruscheinsky MA, Timmons $\mathrm{BC}$, Mahendroo MS. Cervical remodeling during pregnancy and parturition: molecular characterization of the softening phase in mice. Reproduction 2007;134(2):327-40.

8. Kousoulis AA, Ioakeim-Ioannidou M, Economopoulos KP. Access to health for refugees in Greece: lessons in inequalities. Int J Equity Health 2016;15(1):122.

9. Dopfer C, Vakilzadeh A, Happle C, Kleinert E. Pregnancy Related Health Care Needs in Refugees-A Current Three Center Experience in Europe. 2018;15(9).

10. Heslehurst N, Brown H, Pemu A, Coleman H, Rankin J. Perinatal health outcomes and care among asylum seekers and refugees: a systematic review of systematic reviews. BMC Med 2018;16(1):89.
11. Gibson-Helm M, Teede H, Block A, et al. Maternal health and pregnancy outcomes among women of refugee background from African countries: a retrospective, observational study in Australia. BMC Pregnancy Childbirth 2014;14(392.

12. Ali S, Skirton H. Integrative review of cervical cancer screening in Western Asian and Middle Eastern Arab countries. 2017.

13. Khan S, Woolhead G. Perspectives on cervical cancer screening among educated Muslim women in Dubai (the UAE): a qualitative study. BMC Womens Health 2015;15(90.

14. Abboud S, De Penning E, Brawner BM, Menon U, Glanz K, Sommers MS. Cervical Cancer Screening Among Arab Women in the United States: An Integrative Review. Oncol Nurs Forum 2017;44(1):E20-e33.

15. Korukcu 0, Aydin R, Conway J, Kukulu K. Motherhood in the shade of migration: A qualitative study of the experience of Syrian refugee mothers living in Turkey. Nurs Health Sci 2017.

16. Ahmed A, Bowen A. Maternal depression in Syrian refugee women recently moved to Canada: a preliminary study. 2017;17(1):240.

17. Dennis CL, Merry L, Gagnon AJ. Postpartum depression risk factors among recent refugee, asylum-seeking, non-refugee immigrant, and Canadian-born women: results from a prospective cohort study. Soc Psychiatry Psychiatr Epidemiol 2017;52(4):411-22.

18. O'Mahony J, Donnelly T. Immigrant and refugee women's post-partum depression help-seeking experiences and access to care: a review and analysis of the literature. J Psychiatr Ment Health Nurs 2010;17(10):917-28.

Received 4-5-2019

Revised 18-5-2019

Accepted 2-6-2019 